\title{
Ambiente de visualização de dados sociais (ADVS): Uma proposta para apoiar a gestão de stakeholders.
}

\author{
Edson Mota ${ }^{1}$, Paulo C. da Silva ${ }^{1}$ \\ ${ }^{1}$ Programa de Pós-Graduação em Sistemas e Computação, Universidade Salvador - \\ UNIFACS, Salvador (Bahia) -Brasil. \\ edsonmottac@gmail.com, paulo.caetano@pro.unifacs.br
}

\begin{abstract}
The management of stakeholders is a concern inherent in the projects management. Many projects are aborted or not reach his goal in reason of an inefficient management of stakeholders. With the objective of assisting the solution of this question, this article seeks to identify how information technology can contribute to the engagement and construction of sustainable relationships among the stakeholders of a project. For both, it is proposed the confection of a management environment stakeholder called by Viewing Environment of Social Data - VESD, a technological outline that tries to systematize processes, contributing to management and constant improvement of the relationships between the stakeholders and projects.
\end{abstract}

Resumo. A gestão de stakeholders é uma preocupação inerente à gestão de projetos. Muitos projetos são abortados ou não alcançam seu objetivo em razão de uma ineficiente gestão dos stakeholders. Com o objetivo de auxiliar a solução dessa questão, este artigo procura identificar como a tecnologia da informação pode contribuir para o engajamento e construção de relações sustentáveis entre as partes interessadas de um projeto. Para tanto, propõe-se a confecção de um ambiente de gerenciamento de stakeholders denominado de Ambiente de Visualização de Dados Sociais - AVDS, um arcabouço tecnológico que procura sistematizar processos contribuindo para gestão e melhoria constante das relações entre os stakeholders e projetos.

\section{Introdução}

Em 1984, Freeman, uma das primeiras referências a questionar a relevância da análise de partes interessadas no campo das relações entre empresas e sociedade, definiu as partes interessadas ou stakeholders como: "Qualquer grupo ou indivíduo que pode afetar ou ser afetado pela realização dos objetivos de uma empresa." (Freeman 1984). Desde então, o conceito de partes interessadas vem assumindo papeis cada vez mais relevantes em projetos que demandam cada vez maiores habilidades de gerenciamento. $\mathrm{O}$ guia de gerenciamento de projeto $\mathrm{PMBOK}$ define as partes interessadas como: "Pessoas e organizações, tais como, clientes, patrocinadores, a organização executora e o público que estão ativamente envolvidas no projeto ou cujos interesses podem ser positiva ou negativamente afetados pela execução ou pelo término do mesmo." (PMI 2008). 
Por certo, entender este cenário não é uma tarefa trivial. A gestão de stakeholders busca proporcionar um ambiente favorável para que as negociações acerca dos objetivos de um projeto se façam atendendo critérios de transparência e ganhos mútuos, envolvendo no cerne das discussões todos que impactem ou sofram os impactos das decisões tomadas em um projeto.

Entre os métodos para descrever este cenário, pode-se destacar o mapeamento e a análise de partes interessadas que objetivam, fundamentalmente, descrever as relações entre os stakeholders e os projetos e com outros stakeholders (PMI 2008). O entendimento acerca das relações de influência das partes interessadas frente aos objetivos dos projetos, favorece a tomada de decisão, (Schiffer, E., Hartwich F. e Monge M 2010) ao passo que, oferece aos gestores uma visão holística dos riscos e oportunidades contidas nestas relações.

Entretanto, entender as relações entre os indivíduos envolvidos em um determinado projeto, seu posicionamento, interesse, ou mesmo a propensão de um stakeholder em contribuir para o projeto pode ser significativamente complexo. A quantidade de pessoas e suas características se inter-relacionam formando um ambiente de rede que se expande, tornando, muitas vezes, a análise destas relações algo impraticável sem o auxílio de uma ferramenta computacional.

Diante desta dificuldade, propõe-se neste trabalho, a criação do Ambiente de Visualização de Dados Sociais - AVDS, um arcabouço tecnológico que procura, através de uma estrutura integrada de processos, garantir maior entendimento acerca das partes interessadas e seu posicionamento frente aos objetivos de um projeto. Concomitantemente, esse ambiente para visualização dos dados procura contribuir para relações sustentáveis com base em conceitos amplamente aceitos como responsabilidade social corporativa (Banco Mundial 2002) e desenvolvimento sustentável (WCED 1987), inferindo-se que este pode ser aplicado ao gerenciamento de projeto nos mais diversos domínios, inclusive na engenharia de software.

Além da introdução, o artigo está dividido da seguinte forma: A Seção 2 aborda alguns conceitos de Social Network Analysis - SNA na perspectiva da Gestão de Stakeholders, discutindo-se como o ambiente de visualização de dados sociais, proposto neste artigo, pode contribuir para o sucesso no gerenciamento de partes interessadas. Subsequentemente, na Seção 3, são abordados os meandros da gestão de stakeholders no campo da engenharia de software e sua aderência ao modelo proposto; a Seção 4, apresenta o Ambiente de Visualização de Dados Sociais - AVDS, com uma definição detalhada acerca de suas camadas e processos. Na Seção 5, são relatadas as conclusões e, por fim, na Seção 6 são descritas as referências utilizadas na confecção deste trabalho.

\section{Análise de Stakeholders com Social Network Analysis}

Social Network Analysis (SNA) teve sua origem na Sociologia (Mohan, V.R.M. e Palia, A.R. 2013 apud Simmel, 1950) e Antropologia (Mitchell 1969; Boissevain 1974). A abordagem feita em SNA centra-se nas relações entre os indivíduos e procura espelhar questões sobre a representação das dinâmicas e estruturas sociais em um dado cenário de aplicação (Loosemor 1996). 
Mitchell, definiu o conceito de análise de redes sociais como um conjunto específico de ligações entre pessoas, com propriedades adicionais que determinam as características das interligações (Mitchell 1969). Analisando este conceito em relação a análise de partes interessadas, observa-se certa adequação no que se refere aos esforços direcionados para interpretar o comportamento social de um conjunto de indivíduos.

Sob o aspecto cognitivo, Ware ressalta que o poder da visualização gráfica potencializa a capacidade de analisar estruturas complexas representadas no mundo real, mais do que por meio da comunicação verbal (Ware 2005). Meyer complementa citando que os formatos gráficos têm a capacidade de simplificar os aspectos complexos ou dimensionais de um determinado tema, argumentando que as habilidades cognitivas são mais visíveis quando expostas a este tipo de informação do que quando submetidos a números, textos ou argumentações verbais (Meyer 1991).

Assim, a adoção da Social Network Analysis ou SNA para concepção visual das redes de relações entre os stakeholders e sua integração no Ambiente de Visualização de Dados Sociais - AVDS, proposto neste artigo, procura representar as dinâmicas sociais em torno de um projeto, de modo que, o contínuo acompanhamento destas relações, principalmente, as que evidenciam influências ou fluxos de conhecimento entre os stakeholders mapeados, possam contribuir para o melhor desempenho das equipes e, consequentemente, para sucesso dos projetos. A Figura 1 ilustra a representação das relações entre poder e influência dos participantes e como estas poderão ser apresentadas no ambiente de visualização de dados sociais.

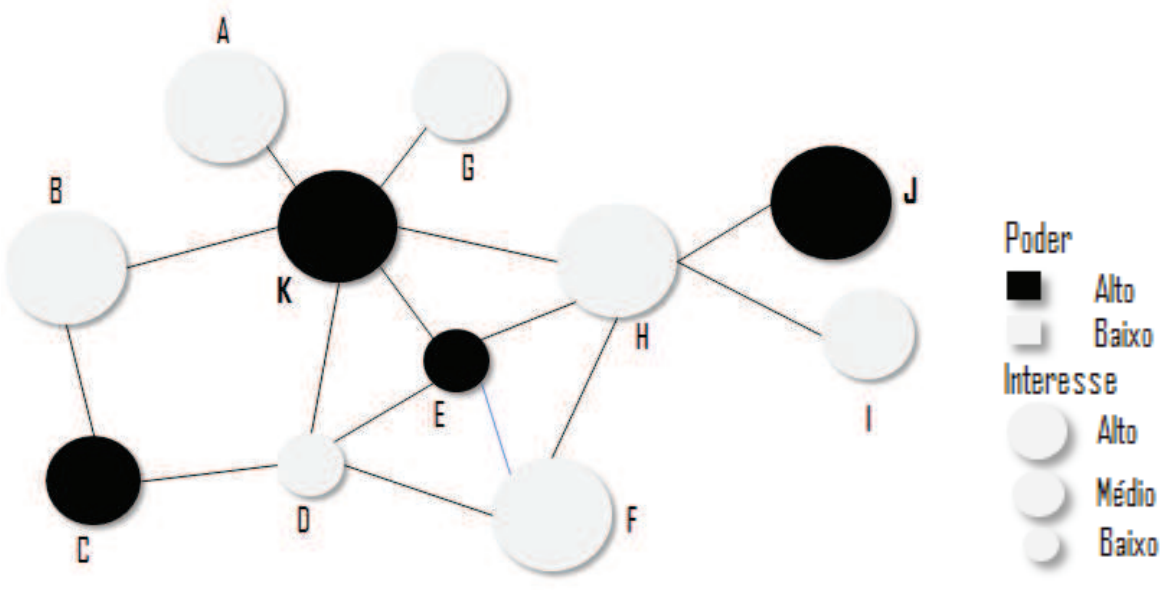

Figura 1. Rede de stakeholders e suas relações.

Analisando a rede ilustrada na Figura 1, é possível perceber que o stakeholder "K" apresenta as características de alto interesse e alta influência e, embora esta característica também seja encontrada no stakeholder "J", sua posição na rede mostra que se trata de um stakeholder mais relevante, uma vez que, este possui além de alto poder e interesse, maior influência dentro da rede de relações, já que se relaciona com um número maior de outros stakeholders. A combinação de poder e interesse alto classifica um determinado stakeholder como crítico para o projeto (PMI, 2008). A análise de sua influência na rede permite detalhar esta criticidade, priorizando os stakeholders frente a sua efetiva capacidade de impactar no projeto. A ideia de mapear 
os stakeholders, de acordo com a sua influência, vem sendo utilizada por diferentes autores (Bourne e Walker, 2005).

Deste modo, observa-se que adotar os conceitos de SNA para compor um ambiente de análise visual de dados acerca dos stakeholders, pode fornecer aos gerentes de projeto um panorama holístico das relações e características dos mesmos em relação à realidade de um projeto. Uma estrutura formal, baseada em grafos, permite a identificação rápida de padrões contidos nas relações entre os stakeholders, que podem, através de suas representações, fornecer um amplo suporte à tomada de decisão, utilizando-se de análises e medidas como: Centralidade, que permite analisar a influência de um nó em determinada rede (Ehrlich e Carboni 2005); Tamanho de uma rede ou medidas de densidade, sendo estas, apenas algumas das especificações abordadas nos conceitos de SNA. Destarte, o ambiente proposto neste trabalho, AVDS, será baseado em Social Network Analysis.

\section{Utilizando a Gestão de Stakeholders na Engenharia de Software}

O gerenciamento de projetos tem um papel relevante no processo de construção de softwares. Assim como as áreas de testes ou engenharia de requisitos, o gerenciamento de projetos procura mitigar os riscos e promover os meios para alcançar os objetivos almejados.

Todavia, por natureza, um projeto é único, e apesar desta diferença ser em grande parte definida pela conformidade dos resultados, escolhas e decisões tomadas acerca de aspectos tecnológicos ou metodologias, o fator determinante para fazer com que um projeto não se repita são as pessoas (Boehm, Barry W. e Rony Ross 1989). Fato que remete ao cerne da questão em discussão no presente trabalho, a relevância das partes interessadas para o sucesso de um projeto.

A gestão de stakeholders no âmbito dos projetos de software pode contribuir significativamente para o aumento da qualidade do produto final. A possibilidade de entender, analisar e gerenciar interesses e expectativas dos envolvidos pode contribuir para um maior engajamento dos interessados aos objetivos do projeto, resultando no aumento da qualidade do projeto e do produto.

Observa-se que o entendimento dos objetivos e regras de negócio em um projeto de software é tarefa crítica para seu sucesso. A engenharia de requisitos aborda metodologias diversas para garantir maior assertividade na coleta de informações que facilitem esse entendimento, todavia, a análise social da rede de relacionamentos entre os stakeholders remete a seguinte questão: "Quem, entre as partes interessadas externas, está mais habilitado para fornecer um dado conjunto de requisitos?" ou ainda, "Quem no conjunto de stakeholders internos está mais habilitado a realizar a coleta e análise dos requisitos de um projeto". Entende-se por stakeholders internos, a própria organização, seus proprietários, diretores, gerentes, funcionários e todos diretamente envolvidos com os objetivos do projeto, já o grupo de stakeholders externos, pode ser constituído por governantes, clientes, comunidade local e todos que afetem ou sejam afetados pelo projeto. (Mitroff 1983) (Canavagh e Mcgovern 1988).

Portanto, a definição dos indivíduos mais adequados a fornecer os requisitos de um sistema ou parte dele, pode ser resultado de um processo de análise de stakeholder, 
no qual o processo de mapeamento, coleta e análise das informações poderá ser realizado com base no Ambiente de Visualização de Dados Sociais - AVDS, proposto neste artigo. Este ambiente permitirá, com base nas características de cada indivíduo, analisar as redes de stakeholders levando em consideração aspectos como interesse, influência, relações e fluxo de conhecimento, frente a um dado domínio, perspectivas que podem auxiliar o gerente de projetos, ampliando sua capacidade de decisão acerca dos indivíduos e seus papeis em um dado projeto.

\section{Ambiente de Visualização de Dados Sociais - AVDS}

Propõe-se neste artigo um modelo para construção do Ambiente de Visualização de Dados Sociais (AVDS). Embora seja uma abordagem tecnológica, propõe-se neste modelo reunir um conjunto de práticas relativas a gestão de stakeholders, buscando reproduzi-lo em um ambiente gráfico, genérico e flexível para visualização de dados sociais. Em seu núcleo de funcionalidades o AVDS procura viabilizar a realização de análises com o fim de responder questões subjetivas acerca das partes interessadas e sua relação com a empresa, com certo grau de consistência e fundamentação.

O ambiente de visualização de dados sociais proposto alinha-se com a gestão de stakeholders, com base em um arcabouço tecnológico que sistematiza os processos de comunicação e gestão de stakeholders. Para isso, são utilizadas abordagens tecnológicas como SNA (Social Network Analysis), fluxos de trabalho e sistemas baseados na web, que deverão constar como alguns dos principais componentes do AVDS.

\subsection{Arquitetura do Ambiente de Visualização de Dados Sociais}

O ambiente proposto está dividido em cinco camadas, além de uma camada conceitual intitulada por boas práticas que tem como objetivo identificar metodologias, técnicas e métodos a serem utilizados no projeto. Esta camada pressupõe as orientações básicas para a construção do ambiente. Essas práticas podem ser adquiridas desde as teorias que enfatizam os princípios da gestão de stakeholder, até os aspectos práticos que envolvem os métodos e apoio ferramental utilizado para realização da tarefa que se pretende que seja orientada com base na boa prática. As demais camadas do ambiente são: (1) Camada de classificação, a qual permite classificar as informações obtidas durante a coleta de dados, considerando questões referentes ao tipo de stakeholder, se interno ou externo, fontes de coleta de dados, informações acerca dos entrevistados; (2) Camada de visualização, que configura o núcleo do modelo, abarcando em sua estrutura os ambientes de visualização de dados sociais baseados em enquadramento e redes, com foco nas análises de interesses, influência e conhecimento, uma estrutura que deve ser adaptada às especificidades do projeto; (3) Camada de comunicação, que procura apoiar-se nos aspectos pertinentes a área de conhecimento de gerenciamento da comunicação para compor um ambiente que abarque o planejamento, execução, monitoramento e controle das comunicações com os stakeholders, através de portais de colaboração; (4) Camada de persistência, Cujo objetivo é garantir a guarda e recuperação das informações. Para isso, utiliza-se um metamodelo baseado em dados representados por XML, denominado XLDM (P. C. da Silva, Times, Ciferri, R.R. e Ciferri, C.D.A 2012) e (P. C. da Silva, Cruz e Times 2011) e, por fim, (5) os Indicadores de engajamento, que consistem em um conjunto de indicadores extraídos das boas práticas da gestão de stakeholder e da responsabilidade social corporativa 
(RSC), que permitem o monitoramento e gestão contínua das relações entre as organizações, seus projetos e as partes interessadas, promovendo os meios para atuar mais ativamente no fortalecimento dessas relações, através do monitoramento das dinâmicas sociais frente as decisões de projeto e seus impactos. Estes indicadores fornecem o feedback necessário para realinhar as estratégias de gerenciamento, de modo a mitigar resistências e gerenciar riscos ao longo da execução ou término de um projeto. A Figura 2 apresenta a arquitetura do ambiente proposto.

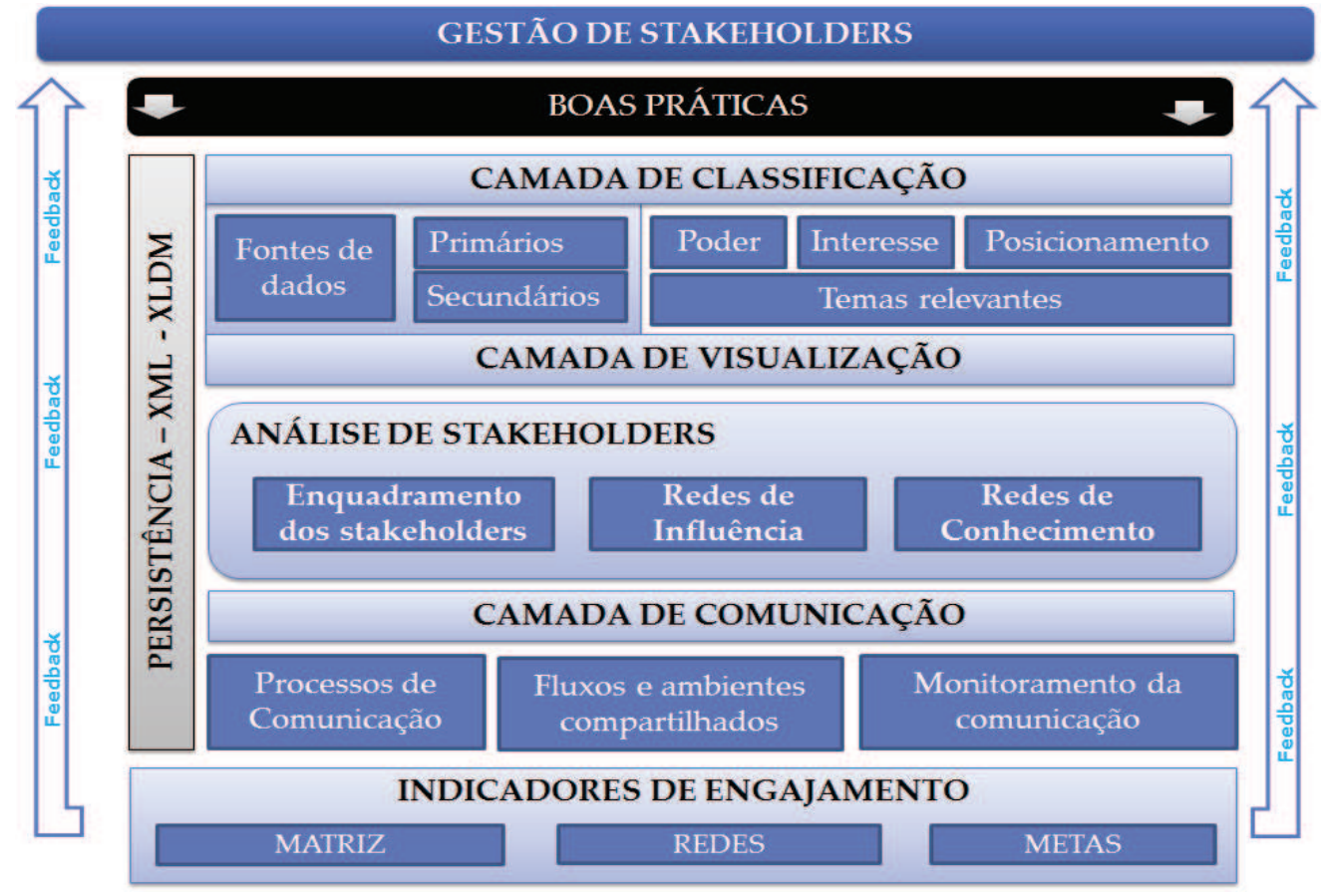

Figura 2. Ambiente de Visualização de Dados Sociais

As seções seguintes descrevem as demais camadas e sua função no AVDS.

\subsubsection{Camada de Classificação}

A camada de classificação consiste de um repositório para o recebimento das informações acerca de cada parte interessada, busca-se com esta camada sistematizar os processos de mapeamento de partes interessadas através da construção de um roteiro de entrada de dados que permita não só a estruturação das informações acerca de um determinado stakeholder, mas também, que forneça informações sobre novas partes interessadas relacionadas à primeira e que não tenham sido mapeadas.

Desta forma, espera-se fornecer o suporte tecnológico a algumas das etapas mais importantes do processo de gerenciamento de stakeholders: a identificação, mapeamento e classificação, mitigando as chances de que uma parte interessadas relevante, ou mesmo com eventual propensão a ascender a papéis mais relevantes, seja ignorada, situação que quando ocorre eleva substancialmente o grau de risco no projeto.

As informações acerca de uma determinada parte interessada poderão ser obtidas através de fontes distintas, classificadas em duas categorias, sendo elas (1) fontes de dados primários, que consistem nas fontes com elevada confiabilidade, como entrevistas com o indivíduo ou um representante de uma entidade; (2) fontes de dados 
secundários, que consistem nos dados obtidos de fontes menos confiáveis, como internet, redes sociais, jornais, revistas.

Subsequentemente, procura-se agrupar os indivíduos com base em um conjunto coerente de características que permita, posteriormente, avaliar o grau de influência de cada um, frente ao alcance dos objetivos do projeto. Embora as análises imponham certo grau de subjetividade, procura-se nesta etapa enquadrar os indivíduos em uma escala de valores pré-determinada variando de um a cinco. Esta valoração busca estabelecer métricas e pesos que auxiliarão nas avaliações subsequentes. Assim, sugere-se a classificação seguindo os seguintes critérios: (1) Poder, que classifica cada indivíduo numa escala de autoridade frente ao projeto. Tal autoridade pode advir desde uma característica explícita, como um cargo de gerência em um determinado departamento, até estruturas de poder mais subjetivas, a exemplo de uma liderança de bairro ou um líder comunitário, no entanto, para que se caracterize determinado grau de poder, é preciso que este exerça nítida influência sobre outros stakeholders mapeados no projeto. Nesta dimensão, o grau 1 (um) representa baixo poder aumentando proporcionalmente até o grau 5 (cinco); (2) Interesse, busca avaliar os níveis de interesse dos stakeholders no que se refere à conclusão dos objetivos do projeto. Baixo interesse pode sugerir baixa propensão a contribuir para o projeto, por outro lado, alto interesse, quando bem gerenciado, pode constituir um importante aspecto de apoio para o alcance dos objetivos, todavia, a capacidade de um dado stakeholder de alavancar o projeto só seria identificada através da análise combinada do interesse com o seu grau de poder e/ou posicionamento; (3) Posicionamento, nesta análise, procura-se avaliar a posição de uma determinada parte interessada em relação aos objetivos de um projeto, buscando aferir o grau de satisfação da mesma no que se refere ao atendimento as suas demandas e seu envolvimento no processo de construção das soluções e resolução de conflitos. A escala utilizada na aferição do posicionamento varia de 1 (um) que representa um posicionamento positivo ou favorável aos interesses do projeto, passando por um posicionamento indiferente ou neutro representados pelos valores 3 (três) ou 4 (quatro) e o posicionamento de grau 5 (cinco), que indica que a parte interessada é terminantemente contra os interesses do projeto ou determinada decisão; (4) Temas de Interesse, que diferentemente dos mecanismos de avaliação mencionados anteriormente, buscam apenas identificar os assuntos mais pertinentes na visão de cada parte interessada. A recorrência dos temas, aliada as métricas aplicadas durante as avaliações, configura uma importante informação para auxiliar na tomada de decisão em projetos.

Deste modo, esta camada tem como função catalogar os impactos das decisões em projetos frente as suas partes interessadas, fornecendo os meios para que as próximas camadas do modelo atuem efetivamente na combinação destes elementos, contribuindo para a construção do conhecimento sobre a realidade social em análise.

\subsubsection{Camada de Visualização}

A camada de visualização tem como propósito auxiliar no entendimento das dinâmicas sociais, esta camada procura delinear através de representações gráficas o mapeamento de stakeholders, objetivando facilitar o entendimento sobre as características dos envolvidos e seus papéis no contexto social em estudo. 
Fundamentalmente, a camada de visualização procura fornecer suporte aos processos de análise dos stakeholders. Estes processos tratam basicamente do entendimento das relações dos mesmos com o projeto e com outras partes interessadas (PMBOK 2013), além da aplicação de modelos de classificação e enquadramento que permitem a realização de análises pontuais voltadas para o indivíduo e sua influência frente ao projeto.

Entre as formas de representação gráfica especificadas no ambiente de visualização de dados sociais, estão os modelos de enquadramento, que procuram através da reunião de dimensões como poder versus interesse, ou poder versus influência, estabelecer relações com base em uma matriz (PMI 2008), e os ambientes de visualização em redes, que procuram reproduzir na forma de grafos, as relações entre os stakeholders, considerando características, papéis e os aspectos pertinentes ao grau de influência e/ou conhecimento dos stakeholders na rede de relacionamento.

A camada de visualização está subdividida em três ambientes principais, sendo eles:

(1) Enquadramento dos stakeholders, que procura classificar os stakeholders com base em sua influência e os interesses comuns. Entende-se por enquadramento dos stakeholders, o agrupamento dos mesmos com base na análise de suas características obtidas no momento da classificação, criando visões que reproduzem o universo social em estudo. Entre as visões, pode-se destacar: (1) a matriz de poder versus interesse que procura representar em quadrantes a posição de cada stakeholder, no que se refere a correlação entre o seu grau de poder e de interesse (PMI 2008); (2) a matriz autoridade versus posicionamento, que com base na matriz anterior, busca identificar os stakeholders críticos frente a sua propensão a ajudar no projeto; e (3) a matriz de avaliação de engajamento, que procura classificar o grau atual e o grau esperado para o engajamento de cada stakeholder do projeto (PMI 2013), dentre outras visões que buscam elevar o conhecimento sobre o papel de cada parte interessada frente aos objetivos do projeto;

(2) Redes de influência, que objetivam permitir as análises qualitativas e quantitativas das condições de uma rede. Diferentemente das análises focadas apenas no indivíduo e sua classificação, as redes de influência buscam respostas nas relações entre as partes interessadas com a empresa e com outras partes interessadas importantes para o projeto.

Destarte, propõe-se a confecção de ambientes baseados em redes que permitam explicitar as relações existentes entre os stakeholders, possibilitando a identificação dos pontos de influência representados por indivíduos ou instituições através das estruturas de convergência existentes entre os nós de uma determinada rede. As influências são classificadas em dois níveis, (1) influentes diretos, que consistem nos influenciadores naturais, como chefes de um setor, orientador religioso, relações de parentesco, e (2) influentes indiretos, que podem ser, o governo local, jornais, revistas, que tenham relevância para o domínio no qual o projeto é empreitado.

Para a confecção das redes, este ambiente apoia-se nos conceitos de Social Network Analysis (SNA). Sua fundamentação conceitual alinha-se com os objetivos do AVDS, tornando o processo de construção e análise das redes um conjunto sistemático de conceitos e abordagens transparentes ao analista, servindo como um facilitador para o entendimento e contribuindo para as decisões de projetos. 
(3) Redes de conhecimento, mais direcionadas a stakeholders internos, estas análises buscam identificar especialistas em determinado domínio que possam contribuir para os interesses do projeto ou parte dele. Identificar as relações de conhecimento em um grupo de partes interessadas de um projeto pode ser significativamente útil. Características como a multidisciplinaridade dos stakeholders pode tornar difícil a identificação de indivíduos adequados a um determinado papel. Assim, as visões baseadas em fluxos de conhecimento podem auxiliar nestas descobertas e trazer significativos benefícios aos projetos em diferentes domínios.

Uma vantagem notória na utilização das visões baseadas em redes de conhecimento consiste no fato de que as mesmas estão livres das hierarquias organizacionais. Durante os processos de mapeamento e classificação dos stakeholders internos, busca-se observar os fatos relevantes ao projeto, tornando a elaboração da rede uma representação mais próxima da realidade, permitindo a identificação das relações informais que levam a um entendimento mais claro sobre o consequente fluxo de conhecimento entre estes grupos. Estas visões podem trazer grandes benefícios na seleção correta de atores para desempenhar papeis durante a execução de um projeto.

\subsubsection{Camada de Comunicação}

A camada de comunicação busca mitigar os problemas de comunicação comuns em projetos. Trata-se de um ambiente de colaboração. Neste ambiente, gerentes de projeto farão os registros das ocorrências com as partes interessadas, identificando mudanças em seu posicionamento. A variação ou constância no posicionamento poderá fornecer uma visão mais detalhada sobre a satisfação de um determinado envolvido, quanto ao desempenho da empresa e/ou projeto, apresentando tendências e fornecendo feedbacks que conduzam a eventuais ajustes no plano de gerenciamento de stakeholders e no plano de gerenciamento de projetos.

A camada de comunicação é constituída por: (1) Processos de comunicação, que consistem na definição dos canais de comunicação e seus direcionamentos. Os processos de comunicação procuram responder as questões: quem fala com quem, quando e como. Esses processos sistematizam uma estrutura de fluxo que gerencia como se dará a comunicação com as partes interessadas nos diversos níveis do projeto; (2) Fluxos e ambientes compartilhados, que consistem na construção de fluxos de trabalho para atendimento as necessidades específicas do projeto. Esta estrutura fornecerá um ambiente flexível para construção de formulários automáticos que trafeguem entre todos os processos internos necessários ao melhor desempenho de um projeto; (3) Monitoramento das comunicações, que permite acompanhar o desempenho da empresa no que se refere a sua capacidade de resposta às partes interessadas envolvidas no projeto, esta camada procura medir e quantificar a qualidade das ações e o quanto estas repercutem no posicionamento social dos envolvidos.

\subsubsection{Camada de Persistência}

A camada de persistência figura no modelo como um ambiente que perpassa diversos processos e camadas. Esta representação procura evidenciar a capacidade de interação desta camada com os pontos de entrada das demais camadas favorecendo $o$ 
desacoplamento, garantindo maior flexibilidade na aplicação do modelo frente a realidade de cada projeto.

A fim de prover maior flexibilidade e independência para o modelo de persistência, propõe-se a utilização de uma arquitetura baseada em XML para representação dos dados utilizados nos diferentes processos e camadas do AVDS. Fundamentalmente, esta camada será composta por diferentes instâncias de documentos XML, interligados por meio de links, que permitam a navegabilidade entre os documentos e a capacidade de realizar consultas e manipulação dos dados que suportam os grafos, matrizes e outros ambientes gráficos dispostos no AVDS. Contudo, a fim de reduzir a complexidade no que se refere a modelagem e acesso aos dados, propõe-se a utilização de um metamodelo baseado em links, denominado XLDM - XLink Data Metamodel (P. C. da Silva, Times, Ciferri, R.R. e Ciferri, C.D.A 2012) e (P. C. da Silva, Cruz e Times 2011). Sua flexibilidade na representação dos relacionamentos garante a utilização deste modelo em diferentes cenários, além de atuar ativamente na resolução de problemas de ordem sintática, estrutural e semântica acerca da heterogeneidade dos documentos XML manipulados pelo ambiente de visualização de dados sociais (P. C. da Silva, Times, Ciferri, R.R. e Ciferri, C.D.A 2012) e (P. C. da Silva, Cruz e Times 2011).

Deste modo, espera-se que a camada de persistência atue não só com a guarda e recuperação das informações, como na construção de uma base de conhecimento que, em última análise, representa a história de cada projeto, constituindo uma importante e confiável fonte de lições aprendidas a serem aplicadas em outros projetos no futuro.

\subsubsection{Indicadores de Engajamento}

A principal função desta camada é fornecer feedbaks acerca da relação das partes interessadas com o projeto. Neste contexto, a camada conceitual de Boas Práticas, que em última análise, representa os processos de gestão adotados pela organização, pode com base nestes feedbaks ajustar seus processos com o fim de realizar eventuais correções de curso, tornando os indicadores de engajamento e a própria gestão de stakeholders cada vez mais consistentes, ao passo que, o projeto avança rumo a conclusão.

Os indicadores de engajamento estão divididos em três macro-categorias, sendo elas: (1) Matriz, que procura estruturar ambientes visuais baseados em planos cartesianos configurados através do cruzamento das forças: poder, interesse, posicionamento e temas relevantes (2) Redes, que buscam representar os aspectos quantitativos observados nas redes, armazenando históricos que conduzam a determinação de tendências no que se refere à relação da empresa com seus stakeholders (3) Meta, que consiste nos indicadores que reproduzem o status atual e o desejado acerca do nível de engajamento de cada stakeholder. Procura-se auxiliar no monitoramento das ações de engajamento, observando seus impactos nas relações com os stakeholders, contribuir assim, para a melhoria contínua destas relações.

\section{Conclusão}

A forte fundamentação teórica no campo da gestão de stakeholders, as práticas adotadas nas diversas metodologias e a sua sistematização em um ambiente tecnológico 
apropriado, constitui o aspecto mais relevante do um ambiente de visualização de dados sociais para a gestão de stakeholders.

Este trabalho procurou viabilizar a análise dos stakeholders com vista na rede de relações a qual eles pertencem, permitindo analisar aspectos como interesse, influência ou temas de preferência frente a sua realidade social e avaliar como essa dinâmica pode ser utilizada em favor do projeto e da comunidade de envolvidos.

Espera-se que o conhecimento fornecido por este ambiente de rede integrada elevem o grau de entendimento dos gestores sobre seus stakeholders, permitindo a adoção de ações mais consistentes e alinhadas aos objetivos de cada projeto. Do mesmo modo, acredita-se que este ambiente proposto contribuirá para maior assertividade nos processos de engajamento, maximizando as chances de sucesso, através da capacidade de convergir influências, interesses e posicionamentos para um ponto comum, no qual stakeholders dialoguem na busca por interesses mútuos.

O ambiente proposto poderá contribuir para estabelecer uma maior harmonia das relações entre gerentes de projeto, empresas e partes interessadas no projeto. O AVDS poderá possibilitar meios para a construção de abordagens empresariais alinhadas aos interesses de seus stakeholders, considerando os objetivos e metas empresariais em relação aos impactos que as decisões organizacionais podem causar na sociedade, um princípio que alinha-se aos conceitos da responsabilidade social corporativa.

Para trabalhos futuros, pretende-se evoluir o grau de detalhamento das camadas com base em estudos bibliográficos, implementação do ambiente AVDS, além da realização de um estudo de caso, com o fim de garantir a aderência das tecnologias propostas para serem usadas no AVDS ao cenário da gestão de stakeholders.

\section{Referências}

Freeman, R. E. (1984). "Strategic management: a stakeholder approach". Massachusetts: Pitman.

Mohan, V.R.M. \& Palia, A.R. (2013), "Stakeholder Management in Infrastructure/Construction Projects: The Role Of Stakeholder Mapping And Social Network Analysis (SNA)", Akeshkar Research Journal, vol. 15, issue 1, pp 48-61 (Online CQUni EBSCOhost).

Zancan C., Santos P. C. F, Campos V. O. (2012) "As contribuições teóricas da análise de redes sociais (ars) aos estudos organizacionais" Revista Alcance - Eletrônica, Vol. 19 - n. 01 - p. 62-82 - jan./mar. 2012

Simmel, G., (1950). In Wolff, K. (Ed.), the Sociology of George Simmel, The Free Press, New York, NY.

PMI, (2013) "A Guide to Project Management Body of Knowledge", 5th Edition. Newtown Square, Pennsylvania: PMI.

PMI. (2008) "A Guide to the Project Management Body of Knowledge (PMBOK® Guide)", 4th ed. Newtown Square, PA, USA: Project Management Institute. (PMI).

Boissevain, J., (1974). Friends of Friends, Basil Blackwell, Oxford. 
Mitchell, R.K., Agle, B.R. and Wood, D.J. (1997). Toward a theory of stakeholder identification and salience: Defining the principle of whom and what really counts. Academy of Management Review 22: p. 853-886.

Loosemore, M., (1998). Social Network Analysis using Quantitative Tool within na interpretative context to explore the management of construction crisis. Engineering, Construction and Architectural Management, Vol. 5 No 4, p 315- 26.

Ware, E., (2005). Visual Queries: The Foundation of VisualThinking, In: Tergan, S.O., Keller, T. (Eds.), Knowledge and InformationVisualization, Springer- erlag, Berlin.

Meyer, A.D., (1991). Visual Data in Organizational Research. Organization BOEHM, Barry W.; ROSS, Rony. "Theory-W Software Project Management: Principles and Examples". Los Angeles: IEEE Transactions on Software Engineering, 1989, Vol. 15, $n^{\circ}$. 7.Science, p 218-236.

P. C. da Silva. (2003). Explorando Linguagens de Marcação para Representação de Relatórios de Informações Financeiras. Dissertação de Mestrado. Universidade Salvador - Salvador/BA. Disponível em: <http://tede.unifacs.br/tde arquivos/2/TDE-2007-0726T170817Z-94/Publico/Dissertacao\%20Paulo\%20Caetano.pdf>. Acessado em: $21 / 01 / 2014$

Mitroff, I. I. (1993) "Stakeholders of the organizational mind", San Francisco: JosseyBass, 1983.

Cavanagh, G. F.; Mcgovern, A. F. (1988) "Ethical dilemmas in the modern corporation", Prentice Hall: Englewoof Cliffs, 1988.

P. C. da Silva, Times V. C., Ciferri, R.R. e Ciferri, C.D.A (2012) "Analytical Processing Over XML and XLink", International Journal of Data Warehousing and Mining, January-March 2012, Vol. 8, No. 1, pages 52-91 DOI: $10.4018 /$ jdwm.2012010101

P. C. da Silva, Cruz, M. S. H. e Times V. C. (2011) "XLDM: AN XLINK-BASED MULTIDIMENSIONAL METAMODEL", JISTEM - Journal of Information Systems and Technology Management Vol. 8, No. 3, Sept/Dec. 2011, p. 641-662 ISSN online: 1807-1775 DOI: 10.4301/S1807-17752011000300007.

BANCO MUNDIAL. (2002) "Public sector roles in strengthening corporate social responsibility: a baseline study", Washington, 2002. Disponível em: $<\mathrm{http} / / /$ www.observatoritercersector.org/pdf/centre_recursos/3_4_fox_01345.pdf/> Acessado em: 21/01/2014.

WORLD COMMISSION ON ENVIRONMENT AND DEVELOPMENT - WCED. (1987), "Our Common Future", Oxford: Oxford University Press, 1987.

Ehrlich, K. \& Carboni, I. 2005. "Inside Social Network Analysis", IBM Technical Report 05-10.

Schiffer, E., Hartwich F., Monge M. (2010) "Who has Influence in Multistakeholder Governance Systems?” International Food Policy Research Institute (IFPRI). , 2010.

Bourne, L., Walker, D. H. T. (2005). "Visualising and mapping stakeholder influence". Management Decision, 43(5), 649-660. 